\title{
Plasma Membrane GABA Transporters Reside on Distinct Vesicles and Undergo Rapid Regulated Recycling
}

\author{
Scott L. Deken, ${ }^{1}$ Dan Wang, ${ }^{2}$ and Michael W. Quick ${ }^{2}$ \\ ${ }^{1}$ Department of Neurobiology, University of Alabama at Birmingham, Birmingham, Alabama 35294, and ${ }^{2}$ Department of Biological Sciences, University of \\ Southern California, Los Angeles, California 90089
}

\begin{abstract}
Plasma membrane neurotransmitter transporters affect synaptic signaling through transmitter sequestration. Transporters redistribute to and from the plasma membrane, suggesting a role for trafficking in regulating synaptic transmitter levels. One method for controlling transmitter levels would be to regulate transporter redistribution in parallel with transmitter release. Thus, how similar are these processes? We show that the trafficking of the GABA transporter GAT1 resembles the trafficking of neurotransmitter-filled synaptic vesicles: (1) transporters located on the plasma membrane are internalized and reinserted into the plasma membrane on the order of minutes; (2) the rate of recycling is depolarization and calcium dependent; (3) GAT1 internalization is associated with clathrin and dynamin; and (4) intracellular GAT1 is associated with multiple compartments and, more importantly, is found on a distinct class of vesicles. These vesicles are clear, $\sim 50 \mathrm{~nm}$ in diameter, and contain many proteins found on neurotransmitter-containing small synaptic vesicles; however, they appear to lack several traditional small synaptic vesicle proteins, such as synaptophysin and the vesicular GABA transporter. These data provide additional support for the hypothesis that GABA transporters traffic in parallel with neurotransmittercontaining small synaptic vesicles and also raise the possibility that some fraction of vesicles found in GABAergic neurons may not be participating in transmitter release but rather in the rapid regulated redistribution of membrane proteins involved in transmitter uptake.
\end{abstract}

Key words: GAT1; neurotransmitter uptake; protein trafficking; recycling; synapse; synaptic vesicle

\section{Introduction}

At GABAergic synapses, removal of neurotransmitter is mediated in part by the neuronal GABA transporter GAT1. The importance of GAT1 is evidenced by the elongation of postsynaptic currents and behavioral changes associated with its inhibition (for review, see Beckman and Quick, 2000). Because transport rates are relatively slow compared with the time course of receptor-mediated signaling (Mager et al., 1993; Wadiche et al., 1995), transporters are thought to exert their effects in part by acting as diffusion sinks, buffering transmitter away from receptors (Tong and Jahr, 1994; Wadiche et al., 1995). Therefore, altering the transporter number at or near the synapse may play a role in regulating neuronal signaling.

Subcellular redistribution of transporters in neurons and glia is well documented. The data for GAT1, and for some other members of this family, are consistent with the hypothesis that neurons regulate GAT1 surface expression in parallel with extracellular transmitter levels. One mechanism by which neurons could accomplish this task is to link transmitter release with transmitter uptake. Thus, how similar are transporter redistribution and synaptic vesicle recycling? The transporters for GABA

Received 0ct. 31, 2002; revised Dec. 12, 2002; accepted Dec. 18, 2002.

This work was supported by National Institutes of Health Grants DA10509 and MH61468 (M.W.Q.). We thankS. L. Schmid for the wild-type and K44A dynamin constructs and E. M. Lafer for the EGFP-clathrin construct. Wealso thank R. G. Zhai, C. C. Garner, C. D. Gancayco, and W. J. Tyler for their expert assistance during the course of this study.

Correspondence should be addressed to Michael W. Quick, Department of Biological Sciences, HNB 228, University of Southern California, Los Angeles, CA 90089-2520. E-mail: mquick@usc.edu.

Copyright $\odot 2003$ Society for Neuroscience $\quad 0270-6474 / 03 / 231563-06 \$ 15.00 / 0$
(Beckman et al., 1998), glycine (Geerlings et al., 2000), and serotonin (Haase et al., 2001) interact with SNARE (soluble $N$-ethylmaleimide-sensitive factor attached protein receptor) proteins, including syntaxin $1 \mathrm{~A}$ and Munc-18. This interaction causes an increase in transporter surface expression (Deken et al., 2000; Geerlings et al., 2001; Horton and Quick, 2001). The dopamine transporter (DAT) is internalized via clathrin and sorted to recycling or degradative pathways (Daniels and Amara, 1999; Melikian and Buckley, 1999; Saunders et al., 2000); internalized DAT can reappear on the plasma membrane, at least under particular pharmacological manipulations (Carvelli et al., 2002). Furthermore, electron microscopy experiments suggest that intracellular GABA transporters (Barbaresi et al., 2001) and glycine transporters (Geerlings et al., 2001) can be found on vesicles in presynaptic terminals. Although these results suggest many similarities between transporter trafficking and synaptic vesicle recycling, many questions remain. What is the rate of transporter recycling? Is this rate regulated by depolarization in a calciumdependent manner? To which intracellular compartments does GAT1 sort? Is the vesicle on which GAT1 resides the same or different from neurotransmitter-filled synaptic vesicles?

\section{Materials and Methods}

Cell culture and $\left[{ }^{3} \mathrm{H}\right] G A B A$ uptake assays. Hippocampal cultures were prepared from postnatal day 0-3 rats (Beckman et al., 1999). Experiments were performed after $10-14 \mathrm{~d}$ in vitro. $\mathrm{CHO}$ cells were maintained in $\alpha$-MEM supplemented with 5\% FBS, L-glutamine, and penicillinstreptomycin. Transfections were performed using FuGene 6 (Roche, Indianapolis, IN) in Opti-MEM I (Invitrogen, Rockville, MD). The lip- 
id-DNA mixture was incubated with the cells for $24 \mathrm{hr}$. Uptake assays were performed as described previously (Bernstein and Quick, 1999). The final $\left[{ }^{3} \mathrm{H}\right] \mathrm{GABA}$ concentration was $40 \mathrm{~nm}$; the total GABA concentration was $30 \mu \mathrm{M}$. Statistical analyses were performed using SPSS (SPSS, Richboro, PA). Two-sample comparisons were made using $t$ tests; multiple comparisons were made using one-way ANOVAs, followed by Tukey's honestly significant difference post hoc test.

Immunocytochemistry and microscopy. Cells were plated onto poly-Llysine-coated coverslips and fixed with $4 \%$ paraformaldehyde, washed, incubated in blocking solution (10\% horse serum, $2 \%$ bovine serum albumin, and $0.25 \%$ Triton X-100 in PBS), treated with anti-GAT1 (1: 200) antibody, washed, treated with biotinylated secondary antibody (1:250; Santa Cruz Biotechnology, Santa Cruz, CA), and stained using Texas Red (Vector Laboratories, Burlingame, CA). Cells were then mounted, sealed with Vectashield (Vector Laboratories), and imaged with a laser-scanning confocal microscope (Olympus Fluoview; Olympus Optical, Mellville, NY).

Biotinylation experiments. Biotinylation experiments were performed as described previously (Whitworth and Quick, 2001). EZ-link SulfoNHS-SS-Biotin (Pierce, Rockford, IL) was used to biotinylate cell surface proteins, and biotin stripping was performed using 2-mercaptoethanesufonic acid.

Biochemical preparations. Synaptosomes were prepared from rat cortices (Montague et al., 1994). Tissue was homogenized in $0.32 \mathrm{M}$ sucrose and centrifuged at $4200 \times g$ for $2 \mathrm{~min}$. The supernatant fraction was centrifuged at 25,200 $\times g$ for $12 \mathrm{~min}$. The resulting pellet was resuspended in $0.32 \mathrm{M}$ sucrose and spun at $25,200 \times g$ for $12 \mathrm{~min}$. This pellet was then resuspended in HEPESbuffered saline solution (HBSS) and centrifuged at $11,000 \times g$ for $5 \mathrm{~min}$. Synaptosomes were fractionated as described previously (Lim et al., 2001) by lysis in hypotonic media on ice for $45 \mathrm{~min}$ and centrifuged at $2000 \times g$ for $20 \mathrm{~min}$. The resulting pellet was saved as the $\mathrm{P} 1$ fraction, and the supernatant fraction was centrifuged at $32,800 \times g$ for $1 \mathrm{hr}$. The resulting pellet was saved as the $\mathrm{P} 2$ fraction, and the supernatant fraction was centrifuged at $100,000 \times g$ for $2 \mathrm{hr}$. The resulting pellet was saved as the $\mathrm{P} 3$ fraction, and the supernatant fraction was saved as the $\mathrm{S} 3$ fraction.

Flotation and immunoisolation. Flotation and immunoisolation were performed as described previously (Zhai et al., 2001). Lysed synaptosomes were adjusted to $2 \mathrm{M}$ sucrose and loaded underneath a discontinuous sucrose gradient of $1.2,1.0,0.8,0.6$, and $0.3 \mathrm{M}$. The gradient was centrifuged at $350,000 \times g$ for 3 hr. Protein A magnetic beads (Dynabeads; Dynal, Great Neck, NY) were incubated overnight with a goat anti-rabbit or anti-mouse linker IgG (Chemicon, Temecula, CA) at $10 \mu \mathrm{g} / \mathrm{mg}$ beads in borate buffer. Beads were collected, washed with PBS- $0.1 \% \mathrm{BSA}$, and blocked $(0.2$ $\mathrm{M}$ Tris at $\mathrm{pH} 8.5$ and $0.1 \% \mathrm{BSA}$ ) for $4 \mathrm{hr}$ at $37^{\circ} \mathrm{C}$. Linker IgG-coated beads were incubated overnight at $4^{\circ} \mathrm{C}$ with rabbit polyclonal anti-GAT1 antibody or monoclonal anti-synaptophysin antibody at a concentration of $10 \mu \mathrm{g} / \mathrm{mg}$ beads in incubation buffer (PBS at $\mathrm{pH} 7.4,2 \mathrm{~mm}$ EDTA, and 5\% FBS). Primary antibody-coated beads and control linker IgG-coated beads were incubated overnight at $4^{\circ} \mathrm{C}$ with isolated fractions. Beads were collected, and the supernatant fractions were saved as nonbound fractions. The beads were washed five times with incubation buffer and three times with

Clathrin-GFP different from control.
PBS for 10 min each and saved as bound fractions. Bound fractions were diluted to the same total volume as the starting material. For electron microscopy, bead-bound fractions were fixed in either $3 \%$ paraformaldehyde and $1 \%$ glutaraldehyde in $0.1 \mathrm{~m}$ phosphate buffer or $4 \%$ glutaraldehyde and $0.8 \%$ tannic acid in $0.1 \mathrm{~m}$ cacodylate buffer to enhance the fixation. After fixation, the bead fractions were rinsed, postfixed by $1 \%$ OsO4, dehydrated, and embedded (Zhai et al., 2001). Antibodies were from the following sources: GAT1, vesicular GABA transporter, synaptotagmin, and VAMP2 (vesicle-associated membrane protein) were from Chemicon; synaptophysin and syntaxin $1 \mathrm{~A}$ were from Sigma (St. Louis, MO); rab3a was from Santa Cruz Biotechnology; rab11 was from BD Bioscience (Lexington KY); and SV2 was from Calbiochem (San Diego, CA).

\section{Results}

After neurotransmitter release, synaptic vesicle proteins are rapidly internalized via clathrin-mediated endocytosis (Heuser, 1989). To determine whether GAT1 is internalized via this mechanism, we used three approaches. The first approach used
A

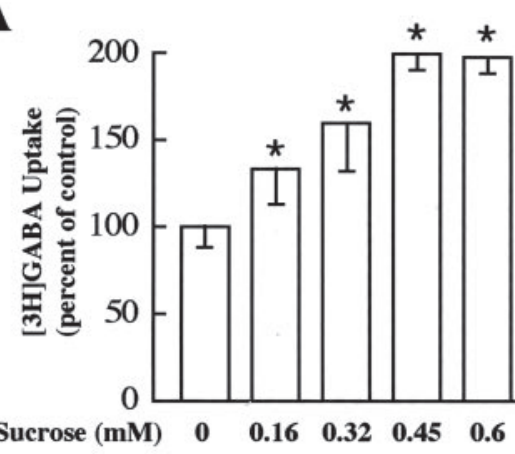

C

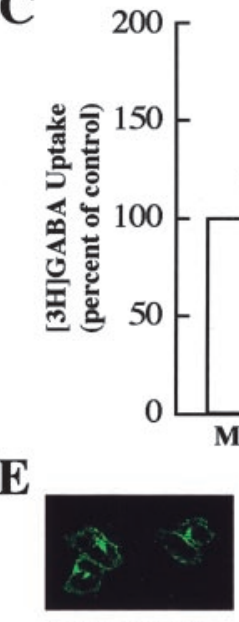

B

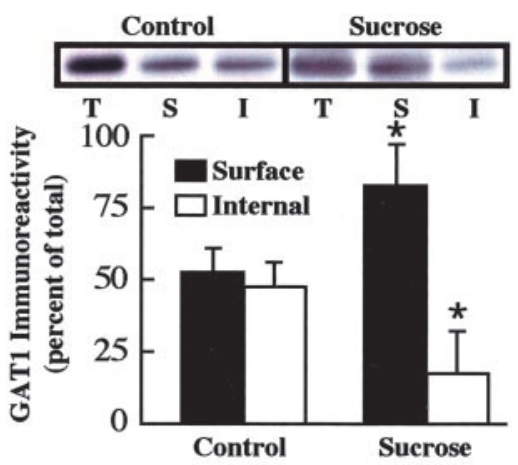

D

Surface

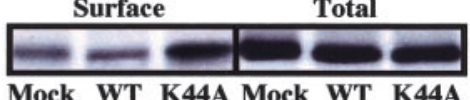

Mock WT K44A Mock WT K44A

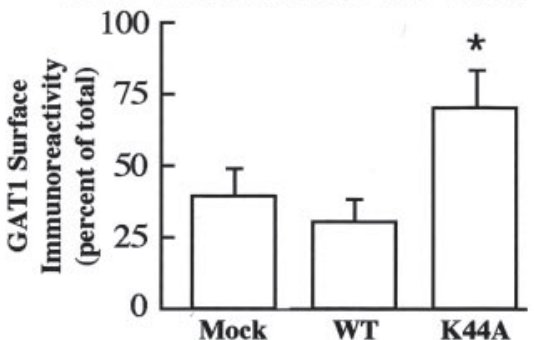

Figure 1. GAT1 internalizes via clathrin. $A$, GABA uptake in hippocampal neurons is increased in the presence of sucrose. Cultures were incubated for 5 min in $90 \mathrm{~mm} \mathrm{~K}^{+}$-HBSS and then incubated with the indicated concentrations of sucrose in $90 \mathrm{~mm}$ $\mathrm{K}^{+}$-HBSS for 5 min before assay. Data are from three experiments, six wells per condition per experiment. Mean GABA uptake under control conditions was $427 \mathrm{fmol} / \mathrm{min}$ per milligram of protein. B, Hypertonic medium increases plasma membrane GAT1 expression. Surface biotinylation of GAT1 was performed in control medium and $5 \mathrm{~min}$ after incubation in medium containing 0.45 m sucrose. Immunoblot shows GAT1 immunoreactivity in total cell lysate ( $T$ ), surface ( $S$; biotinylated), and internal (l; nonbiotinylated) fractions. The graph quantifies GAT1 immunoreactivity in control and $0.45 \mathrm{~m}$ sucrose medium for surface ( filled bars) and internal (open bars) fractions. Data are from three experiments. C, Expression of an inactive dynamin construct increases GABA uptake. CHO cells were cotransfected with vector alone (Mock), wild-type dynamin (WT), or K44A dynamin (K44A) constructs. Data are from two experiments, six wells per condition per experiment. Mean GABA uptake under control conditions was $621 \mathrm{fmol} / \mathrm{min}$ per milligram of protein. $D$, Expression of an inactive dynamin construct increases plasma membrane GAT1 expression. Immunoblot shows surface biotinylation of cells transfected as in C. The graph quantifies results from three separate experiments. E, GAT1 and a clathrin-green fluorescent protein (Clathrin-GFP) construct colocalize in $\mathrm{CHO}$ cells. ${ }^{*} p<0.05$, conditions significantly 
hypertonic media to induce abnormal clathrin polymerization (Heuser and Anderson, 1989). To control for the effect of sucrose on exocytosis ( $\mathrm{Li}$ et al., 2001), we first incubated dissociated hippocampal neurons in high potassium for $15 \mathrm{~min}$ and then added various concentrations of sucrose for $5 \mathrm{~min}$. Treatment with 0.45 and $0.6 \mathrm{M}$ sucrose caused an approximate twofold increase in $\left[{ }^{3} \mathrm{H}\right]$ GABA uptake (Fig. 1 A). This uptake was blocked $94 \%$ by the GAT1-specific inhibitor SKF89976A (data not shown). To determine whether this increase was attributable to increased GAT1 surface levels, we performed surface biotinylation experiments. Treatment of neurons with $0.45 \mathrm{M}$ sucrose increased GAT1 surface immunoreactivity, which correlated with the functional increase (Fig. $1 B$ ). Because the multiple effects of hypertonicity on exocytosis and endocytosis complicate interpretation of these results, our second approach was to determine whether GAT1 internalization was disrupted by expression of an inactive form of dynamin, a protein that participates in clathrin-mediated endocytosis (Damke et al., 1994). We overexpressed the dominant-negative dynamin construct $\mathrm{K} 44 \mathrm{~A}$ in $\mathrm{CHO}$ cells stably expressing GAT1 and examined GABA uptake and GAT1 surface expression. Compared with cells transfected with plasmid vector alone or with wild-type dynamin, cells transfected with K44A dynamin showed increased GABA uptake (Fig. 1C) and increased GAT1 surface immunoreactivity (Fig. 1D). The third approach was to verify colocalization of GAT1 and a fluorescently labeled clathrin construct in $\mathrm{CHO}$ cells (Fig. $1 E$ ). Together, these data suggest that surface GAT1 is internalized via clathrin-mediated endocytosis, consistent with the evidence that dynamin participates in dopamine transporter internalization (Daniels and Amara, 1999; Saunders et al., 2000).

If GAT1 trafficking is similar to that of synaptic vesicle recycling, then one would expect to find GAT1 associated with intracellular recycling compartments (such as endosomes) and vesicle fractions. We used several approaches to test this hypothesis. The first approach used sucrose gradient flotation assays (Fig. $2 \mathrm{~A}$ ). GAT1 associated with organelles of buoyant density similar to the synaptic vesicle protein synaptophysin (Takamori et al., 2000) but not to the cytoplasmic protein Munc-18 (Rowe et al., 2001). To ensure that GAT1 and synaptophysin immunoreactivities were from membrane-bound organelles, we treated synaptosomes with Triton X-100 to disrupt membrane associations (Zhai et al., 2001). The shift to more dense sucrose fractions suggested that these proteins were no longer membrane-associated after detergent treatment.

We next isolated purified organelles from hypotonically lysed synaptosomes (Fig. $2 B$ ). Synaptophysin immunoreactivity was enriched in the P3 fraction, consistent with the idea that the P3 fraction represents the small vesicle fraction. rab5 immunoreactivity was found in the P1 and P2 fractions, supporting the idea that these fractions are enriched in endosomes (Lim et al., 2001). GAT1 was found in all pelleted fractions, consistent with its presence in multiple cellular compartments. We then immunoisolated GAT1 from the P2 fraction. No immunoreactivity was detected in the bound fraction using irrelevant antibodies. Immunoreactivity for the early and recycling endosomal markers rab5 and rab11 and the lysosomal marker lamp2 was detected in GAT1-bound fractions. Immunoreactivity for the $\alpha$-subunit of the $20 \mathrm{~S}$ proteosome was not detected in the GAT1-bound fraction, suggesting that GAT1 is likely degraded by lysosomes and not by proteosomes.

The association of GAT1 with the recycling endosome suggested the hypothesis that the transporter is recycled back to the plasma membrane after internalization. To test this hypothesis
A
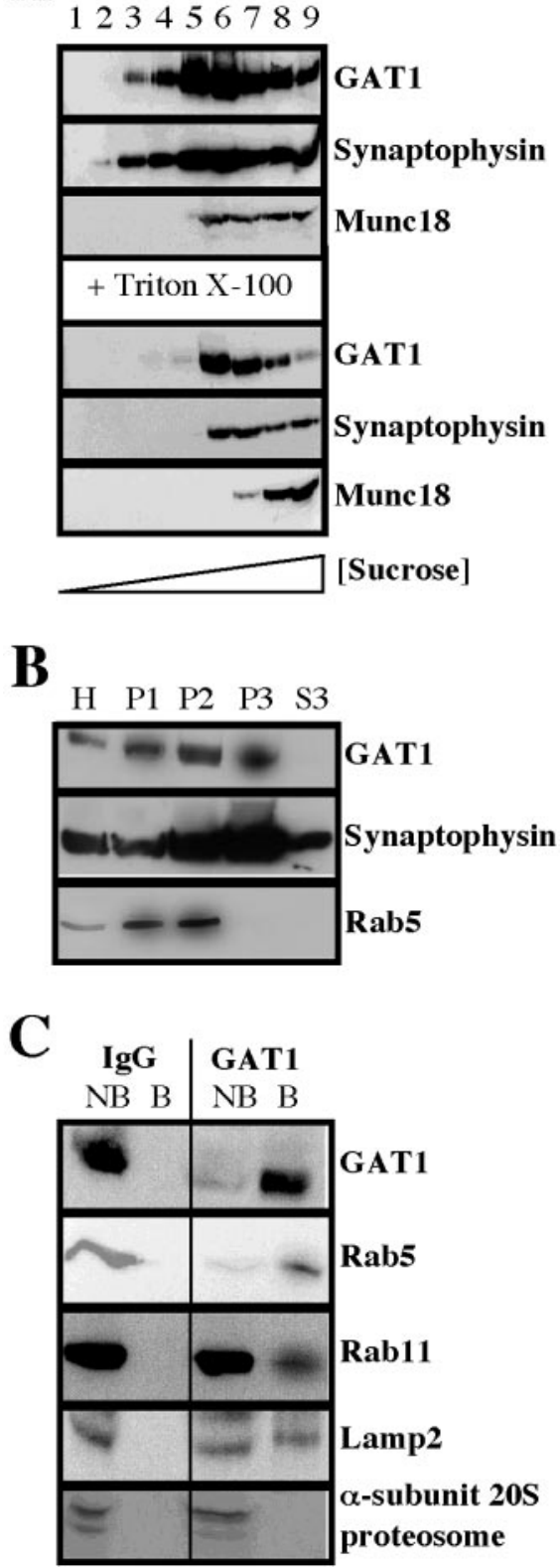

Figure 2. Components of the GAT1 trafficking pathway. A, Intracellular GAT1 has buoyant densities similar to synaptophysin. Shown are immunoblots from a flotation assay of synaptosomes stained with various antibodies. For the membrane disruption experiments, synaptosomes were treated with $2 \%$ Triton X-100 for 45 min before flotation. B, GAT1 is found in multiple cellular compartments based on isolation of purified organelle fractions. C, GAT1 in nerve terminals is associated with both endosomes and lysosomes. Immunoblots are from P2 fractions immunoisolated with beads coated with GAT1 or irrelevant $(I g G)$ antibodies. NB, Nonbound fractions; $B$, bound fractions. Data are representative of at least three experiments.

directly, we performed reversible biotinylation experiments with cleavable biotin molecules. Consistent with previous data (Whitworth and Quick, 2001), after $5 \mathrm{~min}$ at $37^{\circ} \mathrm{C}, \sim 50 \%$ of surface biotinylated GAT1 in neurons was internalized (Fig. $3 A$ ). When the remaining cell surface biotin molecules were stripped and the neurons were incubated at $37^{\circ} \mathrm{C}$ for $5 \mathrm{~min}, \sim 50 \%$ of the internalized, surface-labeled GAT1 returned to the plasma membrane.

To be sure that we were only detecting GAT1 that had resurfaced, we performed several control experiments. First, it was necessary to ensure that the biotin that was being precipitated by 


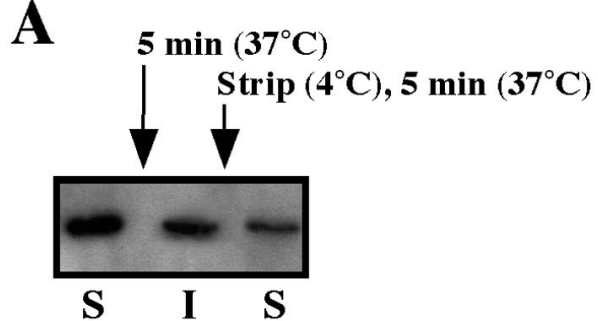

B

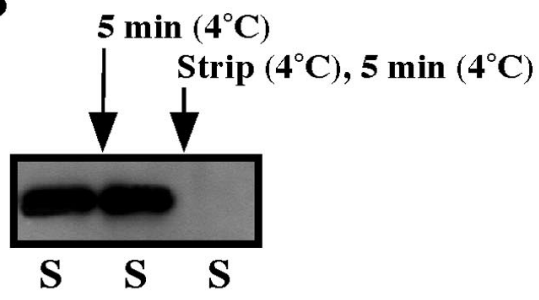

C

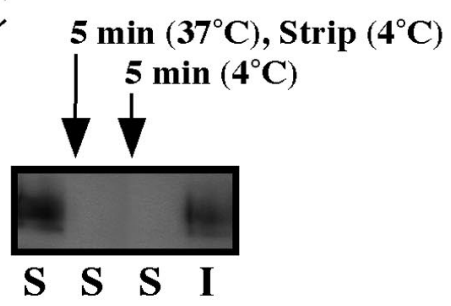

D

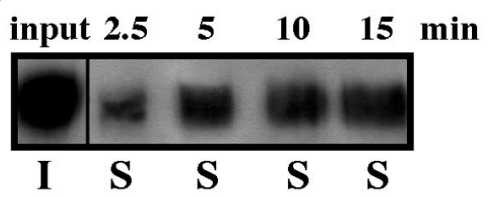

E

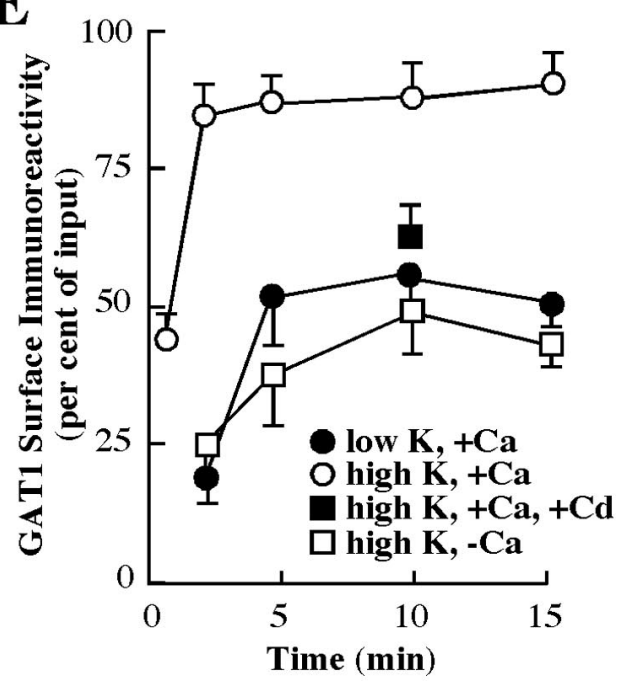

Figure 3. GAT1 recycling rates. $A$, Surface-labeled GAT1 reappears on the plasma membrane. Surface GAT1 (S, left lane) was biotinylated and placed at $37^{\circ} \mathrm{C}$. After $5 \mathrm{~min}$, the amount of internalized GAT1 was determined ( $/$ ). Surface biotin was then removed, cells were placed at $37^{\circ} \mathrm{C}$ for $5 \mathrm{~min}$, and surface GAT1 immunoreactivity was assessed after avidin precipitation ( $S$, right lane). $B$, Surface biotin is completely removed. Surface GAT1 (S, left lane) was biotinylated and reassessed after $5 \mathrm{~min}$ at $4^{\circ} \mathrm{C}(S$, middle lane). Surface biotin was then removed, cells were placed at $4^{\circ} \mathrm{C}$ for $5 \mathrm{~min}$, and surface GAT1 immunoreactivity was assessed after avidin precipitation (S, right lane). C, Avidin added to intact cells only precipitates surface GAT1. Surface GAT1 ( $S$, left lane) was biotinylated and reassessed after biotin removal ( $S$, second lane from left). Avidin was then added to intact cells or to lysed cells, and the resulting surface $(S$, second lane avidin was labeling resurfaced GAT1 only. That is, it was necessary to strip the biotin from surface GAT1 that had not internalized. To verify this, we repeated the experiments at $4^{\circ} \mathrm{C}$ to stop all protein trafficking, and, as expected, GAT1 was not internalized from the plasma membrane (Fig. $3 B$, middle lane). These noninternalized plasma membrane GAT1 proteins could not be precipitated with avidin after biotin stripping, showing that the stripping procedure was efficiently removing the biotin (Fig. $3 B$, right lane). We next verified that the added avidin was only precipitating surface GAT1 molecules. We allowed biotinylated proteins to internalize for $5 \mathrm{~min}$ at $37^{\circ} \mathrm{C}$ and then stripped the remaining cell surface biotin at $4^{\circ} \mathrm{C}$. We were unable to precipitate GAT1 from the cell surface immediately after biotin stripping (Fig. 3C, second lane from left). We then incubated the neurons at $4^{\circ} \mathrm{C}$ for $5 \mathrm{~min}$ and were still unable to precipitate GAT 1 from the plasma membrane (Fig. 3C, third lane from left). However, when we permeabilized the cells, internal biotinylated GAT1 proteins were readily precipitated (Fig. $3 C$, right lane). These control experiments verified that we were precipitating only biotinylated GAT1 that had returned to the cell surface.

We next used this method to determine the rate at which GAT1 recycles by permitting GAT1 internalization and then examining the amount of GAT1 that returned to the surface after various lengths of time (Fig. 3D). A time course for basal GAT1 recycling as a percentage of GAT1 internalization after $5 \mathrm{~min}$ revealed that GAT1 recycling reaches steady state in $\sim 5 \mathrm{~min}$ (Fig. $3 E$ ).

An important related question is whether the recycling of GAT1 is regulated. To test this hypothesis, we determined the rates of GAT1 recycling in the presence of high extracellular $\mathrm{K}^{+}$ concentrations. In the presence of $90 \mathrm{mM} \mathrm{K}^{+}$(or in $30 \mathrm{mM} \mathrm{K}^{+}$; data not shown), both the GAT1 recycling rate and the relative amount of GAT1 on the plasma membrane increased compared with the basal state (Fig. $3 E$ ). This increase in the presence of high $\mathrm{K}^{+}$was prevented by removing $\mathrm{Ca}^{2+}$ from the extracellular medium (in the presence of EDTA) or using $\mathrm{Cd}^{2+}$ to block $\mathrm{Ca}^{2+}$ channels. These data suggest that GAT1 recycling is regulated in a $\mathrm{Ca}^{2+}$-dependent manner.

The data in Figure $2 B$ showed that GAT1 immunoreactivity was found in fractions enriched in vesicles, consistent with electron microscopic data showing GAT1 on vesicles in presynaptic terminals (Barbaresi et al., 2001). To determine the molecular identity of these vesicles, we separated vesicles within the P3 fraction by immunoisolation. We purified both GAT1-containing vesicles and synaptophysin-containing vesicles with specific GAT1 and synaptophysin antibodies conjugated to magnetic beads. Electron microscopy revealed that GAT1-containing vesicles are clear synaptic vesicles with a diameter of $47 \pm 13 \mathrm{~nm}$, similar to synaptophysin-containing vesicles (Fig. 4A). The size of these vesicles was normally distributed, suggesting the presence of GAT1 on a homogenous population of vesicles.

from right) and intracellular ( / GAT1 immunoreactivity was assessed. D, GAT1 basal recycling reaches steady state in minutes. Cells were treated as in $A$, except the second $37^{\circ} \mathrm{C}$ step proceeded for the times shown above the immunoblot. E, GAT1 recycling is regulated by activity in a calcium-dependent manner. Experiments were performed as in $D$ for cultures untreated ( filled circles; $2 \mathrm{~mm} \mathrm{~K}^{+}$and $2.5 \mathrm{~mm} \mathrm{Ca}^{2+}$ ), treated with high $\mathrm{K}^{+}$(open circles; $90 \mathrm{~mm} \mathrm{~K}^{+}$and $2.5 \mathrm{~mm} \mathrm{Ca}^{2+}$ ), treated with high $\mathrm{K}^{+}$in the presence of calcium channel inhibitor ( filled squares; $90 \mathrm{~mm} \mathrm{~K}^{+}, 2.5 \mathrm{~mm} \mathrm{Ca}^{2+}$, and $200 \mu \mathrm{M}\left(\mathrm{d}^{2+}\right)$, or treated with high $\mathrm{K}^{+}$in the absence of $\mathrm{Ca}^{2+}$ (open squares; $90 \mathrm{~mm} \mathrm{~K}^{+}$and $1 \mathrm{~mm}$ EGTA). Treatments began after $5 \mathrm{~min}$ internalization in control medium. Surface GAT1 immunoreactivity, representing recycled GAT1, is plotted as a percentage of GAT1 internalized in $5 \mathrm{~min}$. Data are from four experiments. Data in high $\mathrm{K}^{+}$ (open circles) were significantly different from control at all time points $(p<0.05)$. 


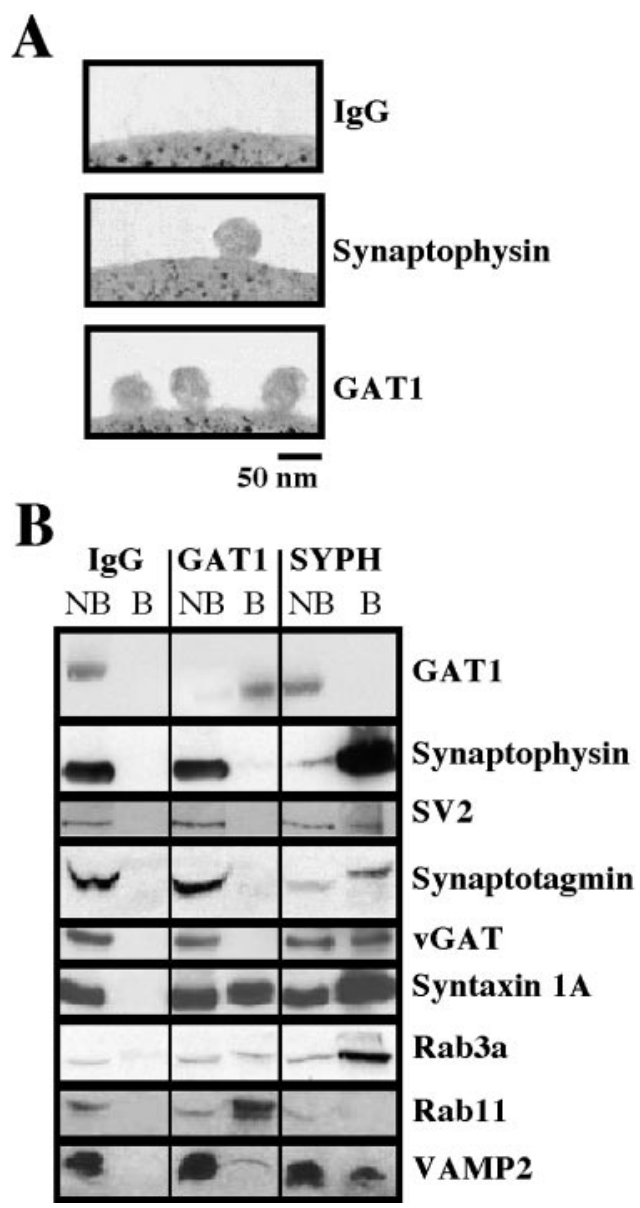

Figure 4. The GAT1-containing vesicle. A, GAT1 and synaptophysin are found on vesicles that are $\sim 50 \mathrm{~nm}$ in diameter. The P3 fraction was separated with GAT1 or irrelevant (IgG) antibodies conjugated to beads and processed for EM. B, The GAT1-containing vesicle is distinct from neurotransmitter-filled synaptic vesicles. The bead-bound $(B)$ fraction and nonbound (NB) supernatant fraction were immunoblotted with various antibodies to proteins known to reside on synaptophysin (SYPH)-containing vesicles. VGAT, Vesicular GABA transporter.

Does GAT1 reside on synaptophysin-positive synaptic vesicles? To test this hypothesis, we examined proteins found on each of the immunoisolated vesicle populations by immunoblot (Fig. $4 B$ ). Our data suggest that the GAT1-containing vesicles are distinct from synaptophysin-containing vesicles. The GAT1containing vesicles appear to contain little or no synaptophysin; synaptophysin-containing vesicles appear to contain no GAT1. In contrast to synaptophysin-containing vesicles, the GAT1containing vesicles appear to lack SV2, synaptotagmin isoforms 1 and 2, and the vesicular GABA transporter. However, the GAT1containing vesicles and synaptophysin-containing vesicles have in common syntaxin 1A, rab3a, and synaptobrevin (VAMP2). GAT1-containing vesicles also contain rab11, suggesting that these vesicles may be derived from the recycling endosome (Ullrich et al., 1996).

\section{Discussion}

The mechanisms by which neurons regulate the release and uptake of neurotransmitter is crucial for understanding synaptic signaling. The present data provide direct evidence that the plasma membrane GABA transporter GAT1 recycles to and from the plasma membrane in neurons through a pathway similar to, and on a time scale comparable with, the recycling of synaptic vesicles (Sudhof, 2000). The rate of recycling is calcium depen- dent, suggesting that the triggers for transmitter release are those that control transporter redistribution. The time scale over which GAT1 recycles supports the hypothesis that neurons regulate transporter expression and neurotransmitter release in parallel (Beckman et al., 1998). One way that GAT1 expression and neurotransmitter-filled synaptic vesicle fusion could be linked mechanistically would be to have plasma membrane transporters expressed on the same vesicles that contain neurotransmitter. However, although we do find that GAT1 resides on a vesicle morphologically similar to neurotransmitter-filled synaptic vesicles, these vesicles are not classic small synaptic vesicles but rather vesicles that likely lack synaptophysin and the vesicular GABA transporter.

Our data showing clathrin-mediated internalization and endosomal sorting is consistent with that shown for DAT, suggesting that this is a pathway shared by this family of plasma membrane transporters (Daniels and Amara, 1999; Melikian and Buckley, 1999; Saunders et al., 2000). We find GAT1 not only resides on endosomes in the nerve terminal but also on a distinct class of synaptic vesicles. These vesicles could represent populations that include endocytic, exocytic, or Golgi-derived vesicles. GAT1 has been shown not to reside on Golgi-derived vesicles containing the active zone assembly proteins piccolo or bassoon (Zhai et al., 2001). These GAT1-positive vesicles may be a cargo vesicle that assembles nonactive zone regions of the synapse.

Neurons control neurotransmitter levels in the synaptic cleft by regulating release (Lin and Scheller, 2000). An emerging view is that neurons can also control neurotransmitter levels by controlling uptake rates (on a time scale of seconds) and transporter surface expression (on a time scale of minutes) (Mennerick et al., 1999; Blakely and Bauman, 2000; Deken et al., 2000; Robinson, 2002). Like neurotransmitter-filled synaptic vesicle recycling, neurons may use transporter recycling as a means of controlling these levels. The present data support the hypothesis that GABA release is linked to its subsequent reuptake. These paths may be similar such that the same factors that regulate release can regulate reuptake. It may be that the transporter resides on a distinct vesicle either because the transporter needs to be recycled at regions distinct from the active zone or because neurons need to fine tune transporter expression independent of release.

\section{References}

Barbaresi P, Gazzanelli G, Malatesta M (2001) $\gamma$-aminobutyric acid transporters in the cat periaqueductal gray: a light and electron microscopic immunocytochemical study. J Comp Neurol 429:337-354.

Beckman ML, Quick MW (2000) The ups and downs of neurotransmitter transporters. The Neuroscientist 6:199-207.

Beckman ML, Bernstein EM, Quick MW (1998) Protein kinase C regulates the interaction between a GABA transporter and syntaxin 1A. J Neurosci 18:6103-6112.

Beckman ML, Bernstein EM, Quick MW (1999) Multiple G-proteincoupled receptors initiate protein kinase $\mathrm{C}$ redistribution of GABA transporters in hippocampal neurons. J Neurosci 19:RC9(1-6).

Bernstein EM, Quick MW (1999) Regulation of gamma-aminobutyric acid (GABA) transporters by extracellular GABA. J Biol Chem 274:889-895.

Blakely RD, Bauman AL (2000) Biogenic amine transporters: regulation in flux. Curr Opin Neurobiol 10:328-336.

Carvelli L, Moron JA, Kahlig KM, Ferrer JV, Sen N, Lechleiter JD, LeebLundberg LM, Merrill G, Lafer EM, Ballou LM, Shippenberg TS, Javitch JA, Galli A (2002) PI3-kinase regulation of dopamine uptake. J Neurochem 81:859-869.

Damke H, Baba T, Warnock DE, Schmid SL (1994) Induction of mutant dynamin specifically blocks endocytic coated vesicle formation. J Cell Biol 127:915-934.

Daniels GM, Amara SG (1999) Regulated trafficking of the human dopa- 
mine transporter. Clathrin-mediated internalization and lysosomal degradation in response to phorbol esters. J Biol Chem 274:35794-35801.

Deken SL, Beckman ML, Boos L, Quick MW (2000) Transport rates of GABA transporters: regulation by the $\mathrm{N}$-terminal domain and syntaxin 1A. Nat Neurosci 3:998-1003.

Geerlings A, Lopez-Corcuera B, Aragon C (2000) Characterization of the interactions between the glycine transporters GLYT1 and GLYT2 and the SNARE protein syntaxin 1A. FEBS Lett 470:51-54.

Geerlings A, Nunez E, Lopez-Corcuera B, Aragon C (2001) Calcium- and syntaxin 1-mediated trafficking of the neuronal glycine transporter GLYT2. J Biol Chem 276:17584-17590.

Haase J, Killian AM, Magnani F, Williams C (2001) Regulation of the serotonin transporter by interacting proteins. Biochem Soc Trans 29:722-728.

Heuser J (1989) The role of coated vesicles in recycling of synaptic vesicle membrane. Cell Biol Int Rep 13:1063-1076.

Heuser JE, Anderson RG (1989) Hypertonic media inhibit receptormediated endocytosis by blocking clathrin-coated pit formation. J Cell Biol 108:389-400.

Horton N, Quick MW (2001) Syntaxin 1A up-regulates GABA transporter expression by subcellular redistribution. Mol Membr Biol 18:39-44.

Li D, Randhawa VK, Patel N, Hayashi M, Klip A (2001) Hyperosmolarity reduces GLUT4 endocytosis and increases its exocytosis from a VAMP2independent pool in 16 muscle cells. J Biol Chem 276:22883-22891.

Lim SN, Bonzelius F, Low SH, Wille H, Weimbs T, Herman GA (2001) Identification of discrete classes of endosome-derived small vesicles as a major cellular pool for recycling membrane proteins. Mol Biol Cell 12:981-995

Lin RC, Scheller RH (2000) Mechanisms of synaptic vesicle exocytosis. Annu Rev Cell Dev Biol 16:19-49.

Mager S, Naeve J, Quick M, Labarca C, Davidson N, Lester HA (1993) Steady states, charge movements, and rates for a cloned GABA transporter expressed in Xenopus oocytes. Neuron 10:177-188.

Melikian HE, Buckley KM (1999) Membrane trafficking regulates the activity of the human dopamine transporter. J Neurosci 19:7699-7710.
Mennerick S, Shen W, Xu W, Benz A, Tanaka K, Shimamoto K, Isenberg KE, Krause JE, Zorumski CF (1999) Substrate turnover by transporters curtails synaptic glutamate transients. J Neurosci 19:9242-9251.

Montague PR, Gancayco CD, Winn MJ, Marchase RB, Friedlander M) (1994) Role of NO production in NMDA receptor-mediated neurotransmitter release in cerebral cortex. Science 263:973-977.

Robinson MB (2002) Regulated trafficking of neurotransmitter transporters: common notes but different melodies. J Neurochem 80:1-11.

Rowe J, Calegari F, Taverna E, Longhi R, Rosa P (2001) Syntaxin 1A is delivered to the apical and basolateral domains of epithelial cells: the role of munc-18 proteins. J Cell Sci 114:3323-3332.

Saunders C, Ferrer JV, Shi L, Chen J, Merrill G, Lamb ME, Leeb-Lundberg LM, Carvelli L, Javitch JA, Galli A (2000) Amphetamine-induced loss of human dopamine transporter activity: an internalization-dependent and cocaine-sensitive mechanism. Proc Natl Acad Sci USA 97:6850-6855.

Sudhof TC (2000) The synaptic vesicle cycle revisited. Neuron 28:317-320.

Takamori S, Riedel D, Jahn R (2000) Immunoisolation of GABA-specific synaptic vesicles defines a functionally distinct subset of synaptic vesicles. J Neurosci 20:4904-4911.

Tong G, Jahr CE (1994) Block of glutamate transporters potentiates postsynaptic excitation. Neuron 13:1195-1203.

Ullrich O, Reinsch S, Urbe S, Zerial M, Parton RG (1996) Rab11 regulates recycling through the pericentriolar recycling endosome. J Cell Biol 135:913-924.

Wadiche JI, Arriza JL, Amara SG, Kavanaugh MP (1995) Kinetics of a human glutamate transporter. Neuron 14:1019-1027.

Whitworth TL, Quick MW (2001) Substrate-induced regulation of gammaaminobutyric acid transporter trafficking requires tyrosine phosphorylation. J Biol Chem 276:42932-42937.

Zhai RG, Vardinon-Friedman H, Cases-Langhoff C, Becker B, Gundelfinger ED, Ziv NE, Garner CC (2001) Assembling the presynaptic active zone: a characterization of an active one precursor vesicle. Neuron 29:131-143. 